\title{
Overexpression of Lin28a Aggravates Psoriasis-Like Phenotype by Regulating the Proliferation and Differentiation of Keratinocytes
}

Soyeon Jang'
Soyoung Jang'
Si-Yong Kim ${ }^{\prime}$
Jiwon Ko'
Eungyung Kim ${ }^{2}$
Ji Yeong Park'
Hyejin Hyung'
Jin Hong Lee'
Su-Geun Lim ${ }^{3}$
Sijun Park $\mathbb{D}^{3}$
Junkoo Yi
Heon-Jin Lee $\mathbb{D D}^{5}$
Myoung Ok Kim ${ }^{2}$
Hyun-Shik Lee
Zae Young Ryoo $\mathbb{D D}^{1}$

'School of Life Sciences, BK2I FOUR KNU Creative BioResearch Group, Kyungpook National University, Daegu, Korea; ${ }^{2}$ Department of Animal Science and Biotechnology, Kyungpook National University, Daegu, Korea; ${ }^{3}$ School of Life Science, Kyungpook National University, Daegu, Korea; ${ }^{4}$ Gyeongsangbukdo Livestock Research Institute, Yeongju, Korea; ${ }^{5}$ Department of Microbiology and Immunology, School of Dentistry, Kyungpook National University, Daegu, 41940, Korea

Correspondence: Zae Young Ryoo; Hyun-Shik Lee

School of Life Science, BK2I FOUR KNU Creative BioResearch Group, Kyungpook National University, Daegu, Korea Tel +82-53-950-736I; +82-53-950-7367 Fax +82-53-943-6925; +82-53-943-2762 Email jaewoong64@knu.ac.kr; leeh@knu.ac.kr
Purpose: Psoriasis is a common and well-studied autoimmune skin disease, which is characterized by plaques. The formation of psoriasis plaques occurs through the hyperproliferation and abnormal differentiation of keratinocytes, infiltration of numerous immune cells into the dermis, increased subepidermal angiogenesis, and various autoimmuneassociated cytokines and chemokines. According to previous research, Lin28 regulates the let-7 family, and let-7b is associated with psoriasis. However, the link between Lin28 and psoriasis is unclear. In this study, an association was identified between Lin28a and psoriasis progression, which promoted the pathological characteristic of psoriasis in epidermal keratinocytes.

Patients and Methods: This study aims to investigate the role of Lin28a and its underlying mechanism in psoriasis through in vivo and in vitro models, which include the Lin28aoverexpressing transgenic (TG) mice and Lin28a-overexpressing human keratinocyte (HaCaT) cell lines, respectively.

Results: In vivo and in vitro results revealed that overexpression of Lin28a downregulated microRNA let-7 expression levels and caused hyperproliferation and abnormal differentiation in keratinocytes. In imiquimod (IMQ)-induced psoriasis-like inflammation, Lin28a overexpressing transgenic (TG) mice exhibited more severe symptoms of psoriasis.

Conclusion: Mechanistically, Lin28a exacerbated psoriasis-like inflammation through the activation of the extracellular-signal-regulated kinase (ERK) and signal transducer and activator of transcription 3 signaling (STAT 3) by targeting proinflammatory cytokine interleukin-6 (IL-6).

Keywords: psoriasis, keratinocyte, Lin28a, let-7

\section{Introduction}

Psoriasis affects approximately $3 \%$ of the global population and is one of the most common and well-researched chronic autoimmune skin diseases. ${ }^{1}$ In addition to its dermatological manifestations, psoriasis significantly impacts the afflicted person's quality of life. $^{2}$ Psoriasis vulgaris, which is referred to as chronic plaque psoriasis, is characterized by well-demarcated scaly erythematous plaques, ${ }^{3}$ which involve any part of the skin; however, it commonly presents on the extensor surfaces (eg, elbows and knees) and scalp. ${ }^{4}$ Psoriasis causes three different processes of cellular alteration in the skin: abnormal differentiation of keratinocytes; hyperproliferation of keratinocytes; and infiltration of immune cells into the dermis and epidermis. ${ }^{5}$ In addition, psoriasis is often associated with systemic inflammation due to the 
production and infiltration of proinflammatory neutrophils and monocytes, which cause comorbidities, which include arthritis, cardiovascular disease, and liver inflammation. ${ }^{6}$ Psoriasis can be triggered by environmental, infectious, and genetic factors, which are stress factors for keratinocytes. These triggers initiate a cascade of events, which include the activation of dendritic cells and differentiation of naive $\mathrm{T}$ cells into $\mathrm{T}$ helper 1 and $\mathrm{T}$ helper 17 cells, which mediate immune responses that are characterized by the release of proinflammatory cytokines. Despite numerous conceptual breakthroughs that have elucidated the pathophysiology of psoriasis, the underlying mechanisms of these pathognomonic changes remain unclear.

Lin28 is an important factor in the regulation of selfrenewal, pluripotency, and the differentiation and development of stem cells. ${ }^{7}$ In addition, Lin28 is an important regulator of microRNA let-7, which is expressed in small quantities in embryonic stem cells that express high levels of Lin28. When the expression of Lin28 decreases during differentiation, the expression of let- 7 increases. ${ }^{8}$ These changes in the expression of Lin28 and let- 7 are the same in most cells and tissues, and embryonic stem cells.

Recent studies demonstrated the potential of microRNAs to regulate gene expression, which might play a role in psoriasis. ${ }^{9}$ MicroRNAs are a group of small non-coding RNAs that can regulate specific gene expression that are involved in diverse biological functions, which include development, lifespan, cell proliferation, differentiation, and signaling pathways. ${ }^{10}$ The microRNA let-7 is one of the first human microRNAs that was identified and is a member of the let-7 family. ${ }^{11}$ Specifically, let-7b is involved in the proliferation and differentiation of neural stem cells. ${ }^{12}$ A recent study demonstrated that let-7b inhibits keratinocyte differentiation by targeting interleukin-6 (IL-6)-dependent extracellular-signal-regulated kinases (ERK1/2) signaling, which might play a role in the pathogenesis of psoriasis. ${ }^{13}$ According to previous research Lin28 downregulates the let-7 family, of which let-7b is a member; however, the link between Lin28 and psoriasis remains unclear.

This study aims to investigate the role of Lin28a and its underlying mechanism in psoriasis through in vivo and in vitro models, which include the Lin28a-overexpressing transgenic (TG) mice and Lin28a-overexpressing human keratinocyte $(\mathrm{HaCaT})$ cell lines, respectively. A previous study illustrated that let-7b did not affect keratinocyte proliferation and apoptosis, ${ }^{13}$ overexpression of Lin28 enhanced cell proliferation in $\mathrm{HaCaT}$ cell lines and induced epidermal hyperproliferation in mice. In the established models, overexpression of Lin28a induced increased proliferation and changes in the differentiation pattern of $\mathrm{HaCaT}$ cells. In addition, Lin28a expression levels were significantly upregulated in psoriasis-induced mouse skin tissue. In imiquimod (IMQ)-induced inflammation, Lin28a-overexpressing TG mice demonstrated severer symptoms of psoriasis, such as epidermal keratinocyte hyperproliferation and immune cell infiltration of the dermis. Lin28a exacerbated psoriasis-like inflammation through the activation of ERK and signal transducer and activator of transcription 3 (STAT3) signaling by targeting proinflammatory cytokine IL-6. In combination, these data uncovered a pivotal role of Lin28a TG mice as a psoriasis mouse model.

\section{Materials and Methods Cell Culture and Transfection}

$\mathrm{HaCaT}$ cells were purchased from AddexBio Technologies (San Diego, CA, USA). HaCaT cells were cultured in Dulbecco's Modified Eagle's Medium (DMEM, Gibco, MA, USA) that was supplemented with $10 \%$ fetal bovine serum (FBS, Gibco) and 1\% penicillin-streptomycin (P/S, Gibco) followed by incubation at $37^{\circ} \mathrm{C}$ in $5 \% \mathrm{CO}_{2}$. To establish stable Lin28a-overexpressing cell lines, cells were seeded into 6-well plates without $\mathrm{P} / \mathrm{S}$. After $24 \mathrm{~h}$, the cells were transfected with pAcGFP-N1 (Mock) or pAcGFP-N1-Lin28a (Lin28a) using the FuGENE HD Transfection Reagent (Promega, WI, USA) following the manufacturer's instructions. HaCaT cells were then treated with $100 \mu \mathrm{g} / \mathrm{mL}$ G418 Sulfate (Gibco) for 7 days to detect successfully transfected cells.

\section{Psoriasis-Like Inflammation Induce by

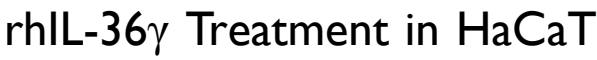

$\mathrm{HaCaT}$ cell lines with stable $\mathrm{f}$ Lin28a overexpression were starved with DMEM/1\% FBS for $24 \mathrm{~h}$ before stimulation. Then, $1 \mu \mathrm{g} / \mathrm{mL}$ recombinant human interleukin-36 $\gamma$ (IL$36 \gamma$, R\&D systems, MN, USA) was treated with Mock and Lin28a. Cells were harvested at 0,6 , and $12 \mathrm{~h}$ after treatment for RNA analysis, and 0,24 , and $48 \mathrm{~h}$ for protein analysis.

\section{Cell Counting Kit-8 Assay}

Cell proliferation was measured using the Cell Counting Kit-8 (CCK-8) assay (Dojindo, MD, USA). Cells were seeded into 96-well plates and incubated for $0,24,48$, 
and $72 \mathrm{~h}$ at $37^{\circ} \mathrm{C}$ in $5 \% \mathrm{CO}_{2}$. Then, $10 \mu \mathrm{L}$ of $\mathrm{CCK}-8$ solution was added to each well, and the cells were incubated for an additional $1 \mathrm{~h}$ at $37^{\circ} \mathrm{C}$ in a $5 \% \mathrm{CO}_{2}$ incubator. The optical density of each well was measured at $450 \mathrm{~nm}$ using a SPECTROstar Nano (BMG LABTECH, Germany).

\section{Mice}

The cytomegalovirus (CMV) promoter was used to generate the whole body Lin28a overexpressing mice. C57BL/ 6 wild-type (WT) and Lin28a TG mice, 8-12 weeks of age, were housed in cages under a strict light/dark cycle (light on at 06:00 $\mathrm{h}$ and off at 18:00 $\mathrm{h}$ ). Genomic DNA was extracted from the tail, and a Lin28a transgene was detected via polymerase chain reaction (PCR) with Lin28a-specific primers. The protocols for all animal experiments followed the National Institutes of Health guidelines for the care and use of laboratory animals and were approved by the Committee for Handling and Use of Animals, Kyungpook National University (Approval No.2020-0117).

\section{IMQ-Induced Mouse Model for Psoriasis}

The mice were treated with a daily topical dose of $62.5 \mathrm{mg}$ of IMQ cream (5\%) that was applied to each shaved back for 5 or 7 consecutive days. Control mice were treated with the same dose of vehicle cream (Vaseline (Vas)) for 5 or 7 days. After treatment for 5 or 7 days, the mice were sacrificed and skin and spleen tissue were obtained.

\section{MicroRNA Reverse Transcription}

Total RNA was extracted from cells or mouse skin tissue using TRIzol reagent (Invitrogen, MA, USA) following the manufacturer's instructions. To measure the microRNA expression level, $10 \mathrm{ng}$ of total RNA were reverse transcribed using TaqMan MicroRNA Reverse Transcription Kit (Applied Biosystems, MA, USA), and subjected to quantitative real-time PCR (qRT-PCR) using TaqMan MicroRNA Assay (Applied Biosystems). qRTPCR was performed to determine the microRNAs expression levels of let-7a, let-7b, and U6. For each sample, the relative expression levels were quantified using the $\Delta \Delta \mathrm{Ct}$ method and were normalized against those of U6.

\section{Immunohistochemistry Analysis}

Mouse skin tissues were fixed with 4\% PFA and embedded in paraffin, and $6 \mu \mathrm{m}$ sections were prepared and stained with Lin28a (1:500, Abcam, UK), keratin 1 (CK1)
(1:1000, Santa Cruz Biotechnology, TX, USA), keratin 10 (CK10) (1:10,000, Abcam), filaggrin (1:1000, Santa Cruz Biotechnology), involucrin (1:1000, Santa Cruz Biotechnology), and $\operatorname{Ki67}$ (1:200, Abcam), which analyzed using immunohistochemistry methods. The VECTASTAIN ABC kit (Vector Labs, CA, USA) was used for anti-rabbit and anti-mouse antibodies. Signals were detected using 3,3'-diaminobenzidinetetrahydrochloride-dihydrate peroxidase substrate (DAB, Vector Labs) staining.

\section{qRT-PCR}

Total RNA was extracted from the cells or mouse skin tissue using a TRIzol reagent (Invitrogen) following the manufacturer's instructions. To measure the mRNA expression level, $1000 \mathrm{ng}$ of total RNA was reverse transcribed using PrimeScript 1st strand cDNA Synthesis Kit (TAKARA, Japan), and subjected to qRT-PCR using TB Green Advantage qPCR Premix (TAKARA). qRT-PCR was performed to determine the expression levels of Lin28a, CK16, CK1, CK10, filaggrin, involucrin, IL-6, interleukin $1 \beta$ (IL$1 \beta)$, IL-36 $\gamma$, tumor necrosis factor $\alpha$ (TNF- $\alpha)$, interleukin17A (IL-17A), S100A9, and $\beta$-actin. For each sample, the relative expression levels were quantified using the $\Delta \Delta \mathrm{Ct}$ method and normalized against those of $\beta$-actin.

\section{Western Blotting}

The total protein was extracted from the cells and mouse skin tissue using PRO-PREP lysis buffer (iNtRON Biotechnology, Korea) following the manufacturer's instructions. Western blotting was performed using primary antibodies against mouse Lin28a (1:2000, Abcam), GFP (1:200, Santa Cruz Biotechnology), CK16 (1:1000, Invitrogen), CK1 (1:1000, Santa Cruz Biotechnology), CK10 (1:10,000, Abcam), filaggrin (1:1000, Santa Cruz Biotechnology), involucrin $(1: 1000$, Santa Cruz Biotechnology), ERK (1:1000, Cell Signaling Technology), phospho-ERK (p-ERK) (1:1000, Cell Signaling Technology), STAT3 (1:2000, Cell Signaling Technology, MA, USA), p-STAT3 (1:2000, Cell Signaling Technology), I $\mathrm{I} \mathrm{B} \alpha \quad(1: 1000$, Cell Signaling Technology), $\quad \mathrm{p}-\mathrm{I} \kappa \mathrm{B} \alpha \quad(1: 1000, \quad$ Cell Signaling Technology), and $\beta$-actin $(1: 10,000$, Santa Cruz Biotechnology).

\section{Statistical Analysis}

All data are presented as the mean \pm SEM. Statistical analyses were performed using GraphPad Prism version 
9.1.2 (GraphPad Software, San Diego, CA, USA). ${ }^{*} p<0.05,{ }^{* *} p<0.01, * * * p<0.001$, and ${ }^{* * * *} p<0.0001$.

\section{Results}

\section{Lin28a Overexpression Promotes the Proliferation and Differentiation of Keratinocytes and Regulates} Psoriasis-Related Genes in $\mathrm{HaCaT}$

To study the function of Lin28 in the human skin epidermis, HaCaT were used for experimentation. First, the human Lin28a plasmid vector (pAcGFP-N1-Lin28a) was transfected in $\mathrm{HaCaT}$. When stable cell lines were established, the mRNA expression level of Lin28a was investigated by RT-PCR. Lin28a mRNA expression levels were approximately 12 times higher in Lin28aoverexpressing cells (Lin28a) than in Mocks (Figure 1A). The expression levels of let-7a and let-7b, which are members of the microRNA let-7 family, significantly decreased in Lin28a-overexpressing cells (Figure 1B). In addition, the result of Western blotting showed higher levels of Lin28a proteins in the Lin28a cell line compared with that of Mocks (Figure 1C). In addition, the CCK- 8 proliferation assay results showed that Lin28a overexpression significantly enhanced HaCaT cell proliferation (Figure 1D). These observations agreed with the results from previous studies where Lin28a promoted cell growth and proliferation by repressing let-7 microRNAs biogenesis. ${ }^{7}$

To investigate the effect of $\operatorname{Lin} 28$ overexpression on keratinocyte differentiation, early and late differentiation markers were confirmed. Early differentiation markers for keratinocytes, CK1, and CK10 increased in Lin28aoverexpressing cells (Figure 1E). In addition, an increase in the keratinocyte late differentiation markers, filaggrin and involucrin, was observed (Figure 1E). Out of the proinflammatory cytokines, IL- 6 , TNF- $\alpha$, and interferongamma (IFN- $\gamma$ ), the expression levels of IL- 6 and TNF- $\alpha$ were enhanced by Lin28a overexpression (Figure 1F). However, there was no difference in the expression level of IFN- $\gamma$ (Figure 1F). Although IL-6, TNF- $\alpha$, and IFN- $\gamma$ are all major cytokines in psoriasis, IL- 6 and TNF- $\alpha$ are mainly secreted from keratinocytes and $\mathrm{T}$ cells during early psoriasis, and IFN- $\gamma$ is mainly secreted from $\mathrm{T}$ cells during late psoriasis. ${ }^{14}$ In combination, the overexpression of Lin28a influenced the proliferation and abnormal differentiation of keratinocytes with a change in cytokine expression.

\section{Expression of Differentiation Markers and Cytokines After IL-36 $\gamma$ Treatment for the Psoriasis-Like Condition in $\mathrm{HaCaT}$}

To investigate the function of Lin28a in the inflammatory condition, cells were stimulated with recombinant human IL-36 $\gamma$, which might trigger psoriasis through the chronic activation of keratinocytes. Psoriasis is usually characterized by hyperproliferation and abnormal differentiation of keratinocytes. ${ }^{15}$ Following cytokine treatment, cells were harvested at specific time points $(0,6$, and $12 \mathrm{~h})$, and the differentiation markers were examined by RT-PCR. At these time points, mRNA expression levels of the early and late differentiation markers were higher in the Lin28aoverexpressing cell line than in Mocks (Figure 2A). These results meant that Lin28a overexpression maintained the expression of abnormal differentiation markers without stimulation and when psoriasis-like conditions were induced. To determine if Lin28a overexpression affected the production of proinflammatory cytokines in the inflammatory condition, the relative mRNA levels of IL- 6 and TNF- $\alpha$ were measured. These results showed that psoriasis-like inflammation that was induced by IL-36 $\gamma$ increased cytokine expression at specific time points. IL-6 expression was highest at $6 \mathrm{~h}$ in Mocks and Lin28a-overexpressing cells, and higher expression was maintained in Lin28aoverexpressing cells. TNF- $\alpha$ showed the highest expression at $12 \mathrm{~h}$, and the expression of TNF- $\alpha$ was higher than that of Mocks in Lin28a-overexpressing cells (Figure 2B). These data implied that the overexpression of Lin28a played a pivotal role in psoriasis-like inflammation.

\section{Time-Dependent Change in Lin28a and Psoriasis-Related Markers in IMQ-Induced Psoriasis-Like Mice}

To identify a link between Lin28 and psoriasis, Lin28a expression in IMQ-induced mice was evaluated. First, IMQ cream, which is commonly used to induce psoriasis in mouse models, ${ }^{16}$ was applied to WT mice for 7 days, then the mice were sacrificed to retrieve skin tissue after 7 days. The relative mRNA expression levels of Lin28a in WT mice increased after IMQ treatment. In addition, the expression level of Lin28a was highest 5 days after IMQ treatment (Figure 3A). MicroRNA let-7a and let-7b expression levels decreased and Lin28a expression increased by psoriasis induction (Figure 3B). Due to the observed correlation between Lin28a and induced 
A

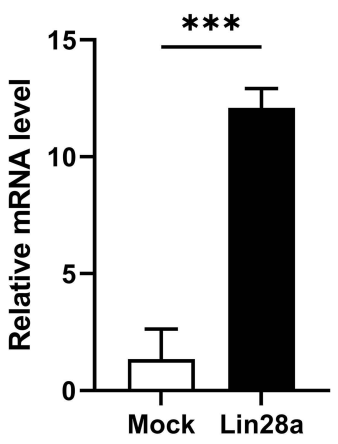

C

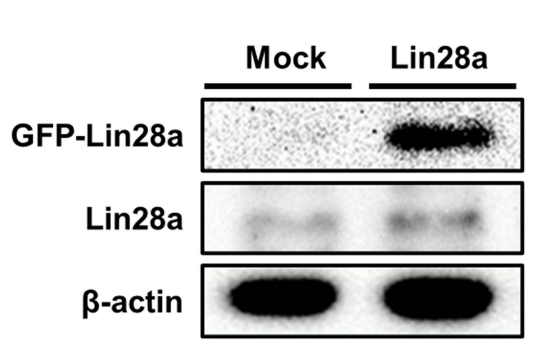

E

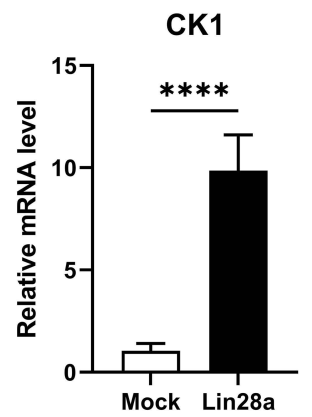

Lin28a

F
B

let-7

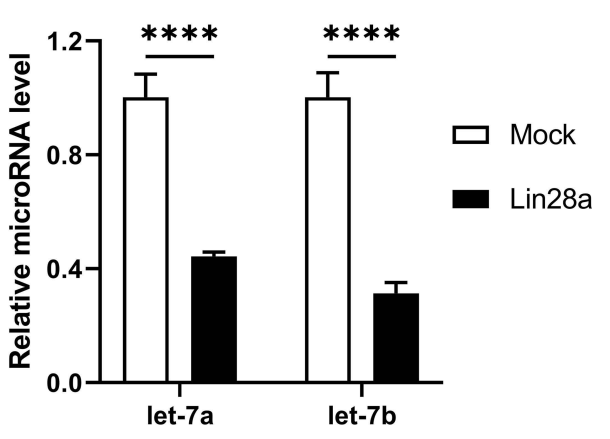

D

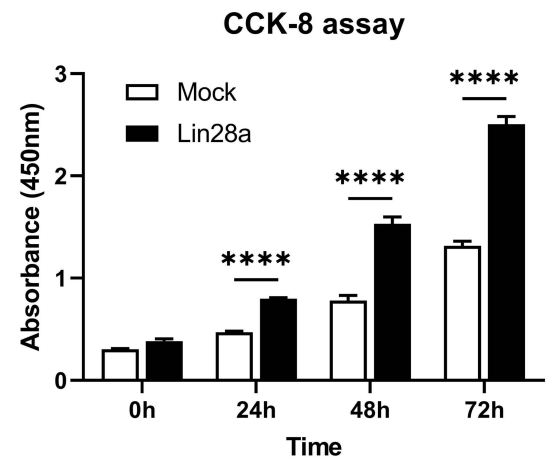

Filaggrin

Involucrin
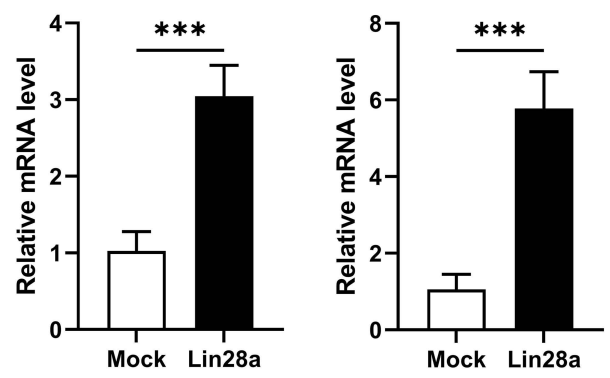

IFNy

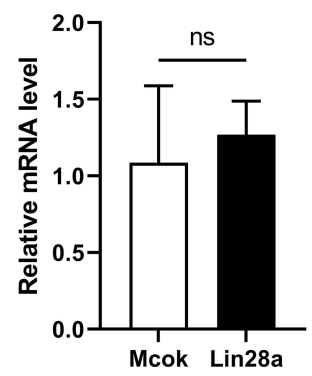

Figure I Lin28a overexpression promotes proliferation and differentiation of keratinocytes and regulates psoriasis-related genes in HaCaT: (A) total RNAs were isolated from Mocks and Lin28a HaCaT cell lines and reverse transcribed. Relative mRNA expression levels of Lin28a were analyzed by RT-PCR; (B) total RNAs were isolated from Mocks and Lin28a HaCaT cell lines and reverse transcribed using TaqMan MicroRNA Reverse Transcription Kit (Applied Biosystems). Relative expression levels of let-7 microRNAs were examined through RTPCR using TaqMan MicroRNA Assay (Applied Biosystems); (C) total proteins were isolated from Mocks and Lin28a HaCaT cell lines. Expression levels of Lin28a-GFP fusion, Lin28a, and $\beta$-actin protein levels were analyzed via Western blotting; (D) proliferation ability was analyzed using CCK-8 assays until 72 h; (E) relative mRNA expression levels of early and late differentiation markers in Mocks and Lin28a HaCaT cell lines were examined through RT-PCR; (F) relative mRNA expression levels of proinflammatory cytokines in Mocks and Lin28a $\mathrm{HaCaT}$ cell lines were examined through RT-PCR. T-tests were performed using GraphPad Prism 9. All data are presented as mean \pm SEM; $* p<0.05$, **** $p<0.00 \mathrm{I}$, and ***** $p<0.000 \mathrm{I}$ compared with Mocks. 


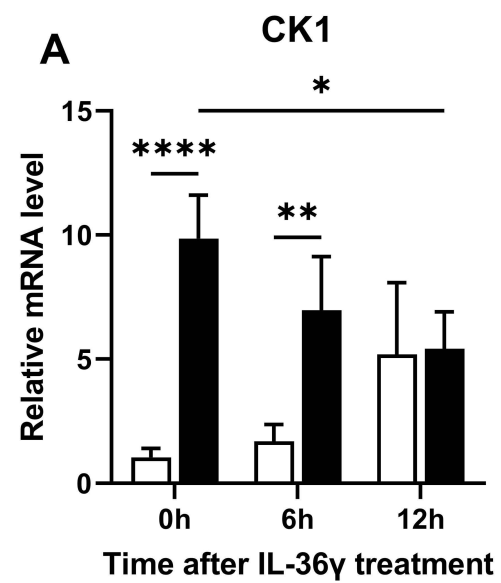

Filaggrin

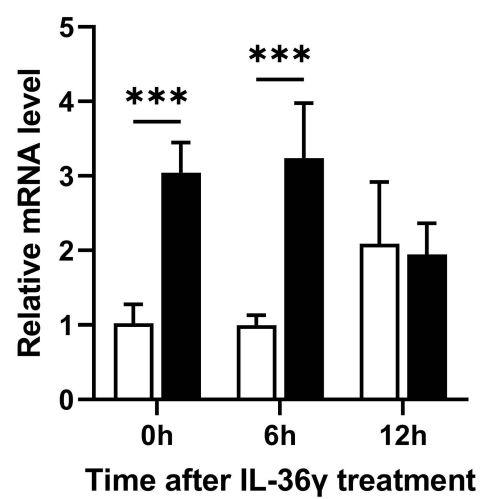

B

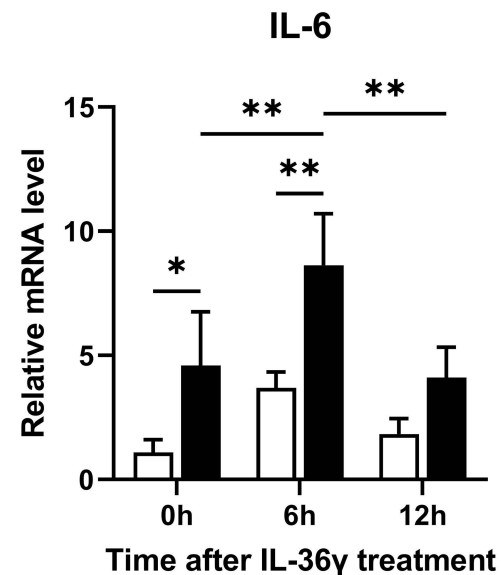

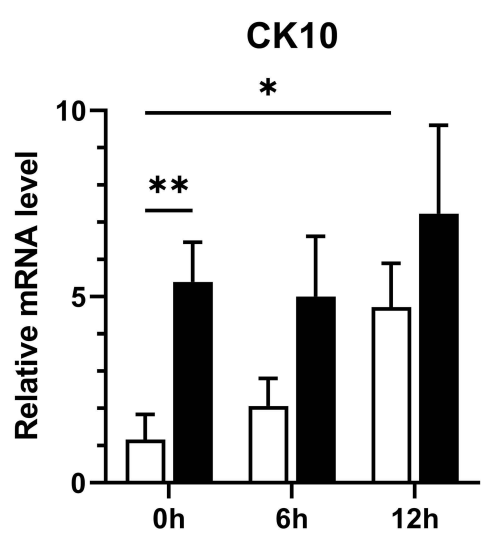

Time after IL-36y treatment

\section{Involucrin}

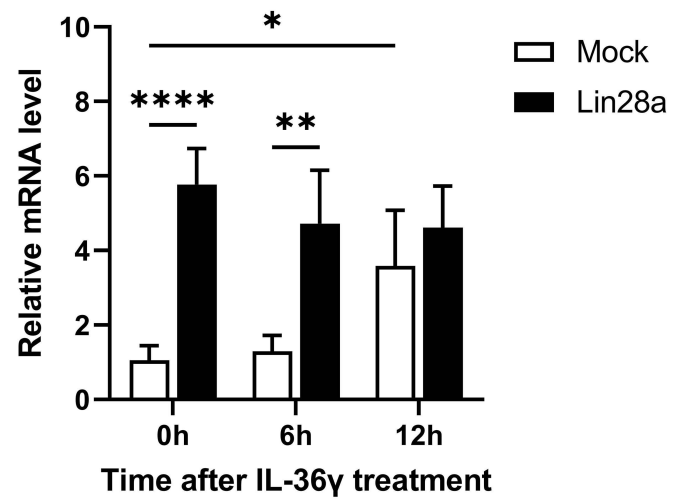

Time after IL-36y treatment

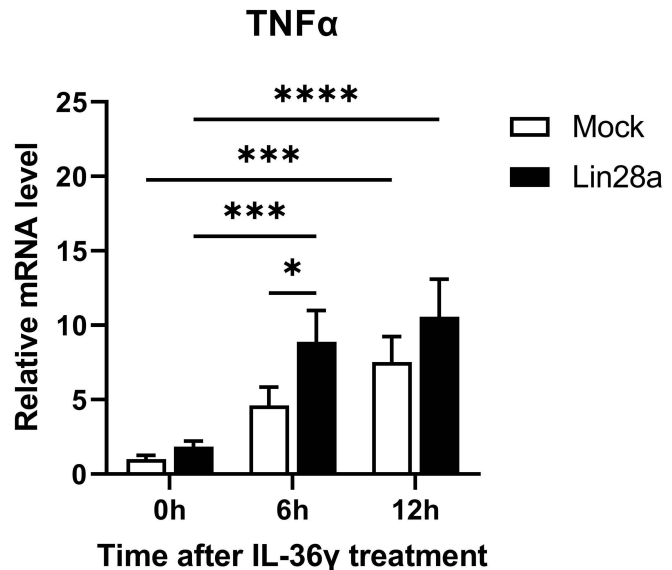

Figure 2 Expression of differentiation markers and cytokines after IL-36 $\gamma$ treatment for the psoriasis-like condition in HaCaT. Mocks and Lin28a-overexpressing HaCaT were treated with recombinant human IL-36 $\gamma$ for 0,6 , and $12 \mathrm{~h}$. Total RNAs were isolated from Mocks and Lin28a HaCaT cell lines treated with IL-36 $\gamma$ and reverse transcribed; (A) relative mRNA expression levels of differentiation markers were measured by RT-PCR; and (B) relative mRNA expression levels of proinflammatory cytokines were examined through RT-PCR. Two-way ANOVA followed by Tukey's multiple comparisons test was performed using GraphPad Prism 9 . All data are presented as mean $\pm \mathrm{SEM} ;{ }^{*} p<0.05,{ }^{* *} p<0.01$, $* * * p<0.001$, and $* * * * p<0.0001$ compared with each group.

psoriasis, psoriasis-related proliferation and differentiation markers were confirmed in mouse skin tissue. The proliferation marker, CK16, significantly increased after psoriasis was induced in mRNA and protein levels. (Figure 3C and D). This result corresponded to previous results that Lin28a promotes cell proliferation and is 


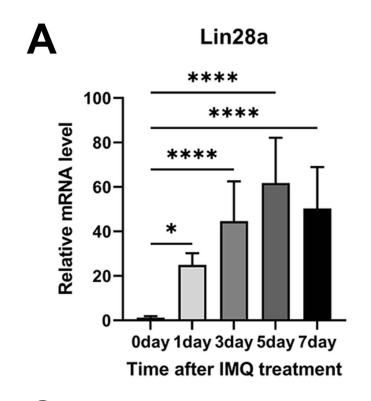

C

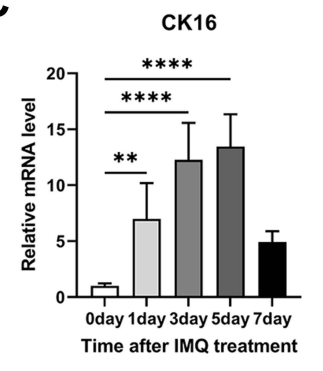

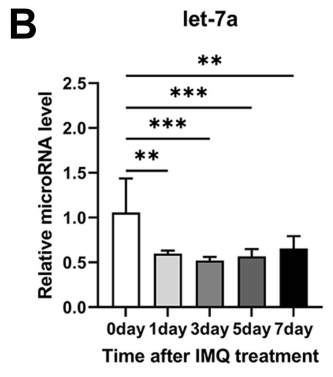

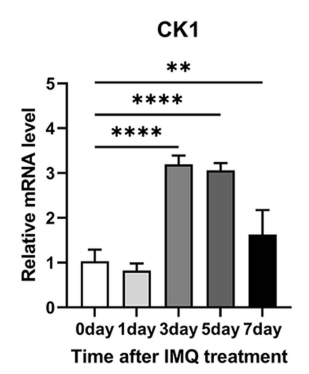

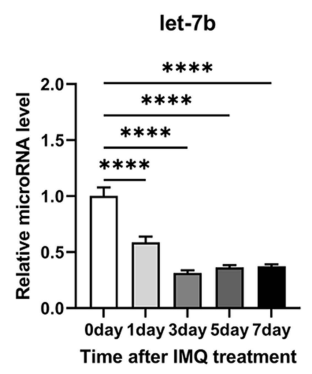

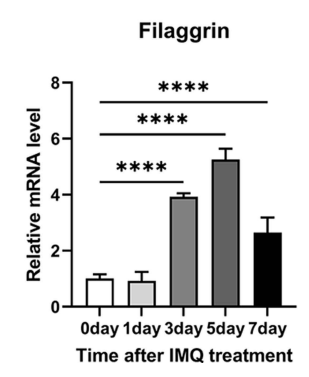

D

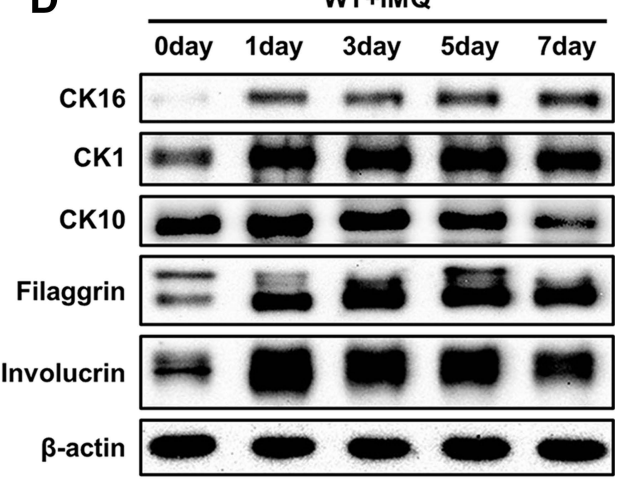

Figure 3 Time-dependent change in Lin28a and psoriasis-related markers in IMQ-induced WT mice. WT mice were treated with a daily topical dose of 62.5 mg of IMQ cream (5\%) for 0, I, 3, 5, and 7 days; (A) total RNAs were isolated from mouse skin tissue and reverse transcribed. Relative mRNA expression patterns of Lin28a were analyzed by RT-PCR; (B) total RNAs were isolated from mouse skin tissue and reverse transcribed using TaqMan MicroRNA Reverse Transcription Kit. Relative expression levels of let-7 microRNAs were measured by RT-PCR using TaqMan MicroRNA Assay; (C) relative mRNA expression level of proliferation marker (CK I6) and differentiation markers (CKI and filaggrin) are shown at specified dates; (D) protein levels of proliferation and differentiation markers in WT mouse skin after IMQ treat using Western blotting. One-way ANOVA followed by Dunnett's multiple comparisons test was performed using GraphPad Prism 9 . All data are presented as mean \pm SEM; ${ }^{*} p<0.05, * * p<$ 0.01 , $* * * p<0.001$ and $* * * * p<0.0001$ as compared with untreated WT mice.

linked to a crucial feature of psoriasis, which is significantly increased proliferation of keratinocytes. ${ }^{7}$ Each of the early and late differentiation markers, CK1 and filaggrin, increased in the mRNA levels following IMQ treatment (Figure 3C). In addition, increased protein levels of the proliferation and differentiation markers were detected (Figure 3D). The expression levels of Lin28a and psoriasis-related markers were highest in day 5 skin from the mice. Based on these results, IMQ treatment was performed for 5 days, and samples were collected on day 6 for use in the following experiments.

\section{Promoted Proliferation of Keratinocytes in Lin28a TG Mice}

To determine the role of Lin28a in epidermal keratinocytes in vivo, TG mice that overexpressed Lin28a under the CMV promoter were studied. Genotyping was performed using PCR to verify the successful generation of Lin28a TG mice (Figure 4A). The Lin28a level was highly expressed in the skin tissue (Figure 4B). Western blotting revealed a higher expression of Lin28a protein in Lin28a TG mice than in WT mice (Figure 4C). In addition, expression levels of let-7a and let-7b microRNAs were lower in Lin28a TG mice (Figure 4D). Lin28a-overexpressing TG mice were developed for these experiments, and 8-12-week-old WT and Lin28 TG mice were used in the subsequent experiments.

To determine the potential role of Lin28a in skin psoriasis, IMQ cream that contained 5\% IMQ was topically applied to the shaved back skin of Lin28 TG and WT mice for 5 consecutive days to induce psoriasis. Although Lin28a TG and WT mice that were treated with IMQ developed erythematous lesions covered with white silver scales, the skin from Lin28 TG mice appeared to be more erythematous than WT without IMQ treatment (Figure 4E). Hematoxylin and eosin (H\&E) staining was performed on mouse skin samples (Figure 4F) and based on this result, the thickness of the epidermis was measured. According to the measurement analysis, the epidermis thickness of Lin28a TG was thicker than that of WT, which agreed with the comparison between the Vas and IMQ-treated groups (Figure 4G). In addition, the $H \& E$ results revealed immune cell infiltration in Lin28a TG and WT mice. Immune cell infiltration was performed by counting stained cells in the dermis. According to these results, untreated Lin28a TG had more infiltration of immune cells than WT mice with or without IMQ, but IMQtreated Lin28a TG mice did not differ from IMQ-treated WT mice (Figure 4H). To account for the thick epidermis thickness of Lin28a TG mice, the expression of proliferation 


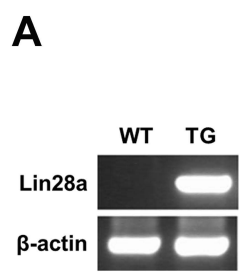

E

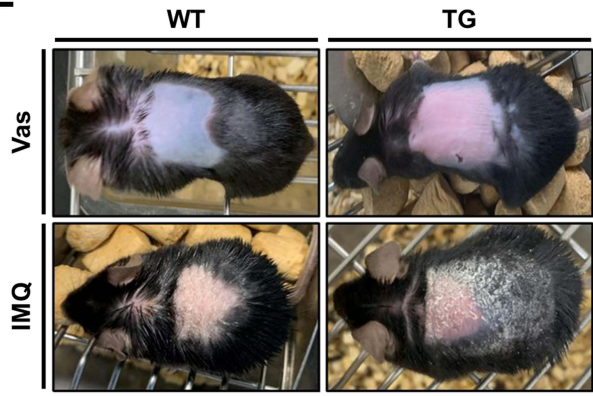

C

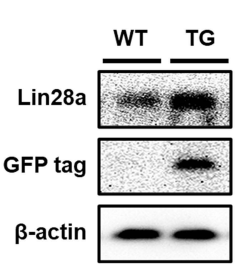

D

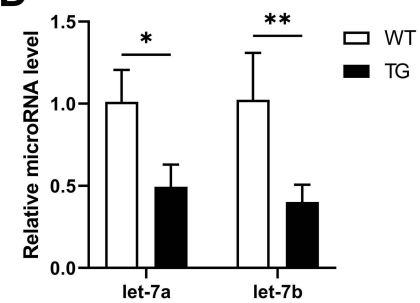

F

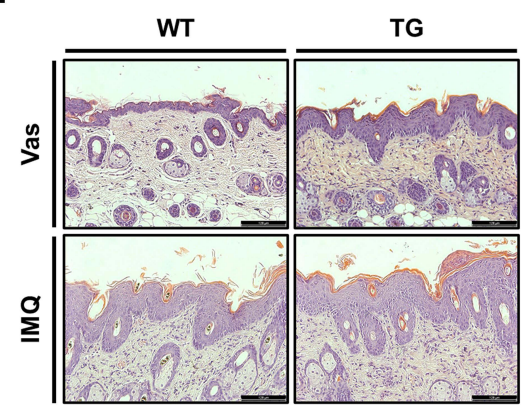

G

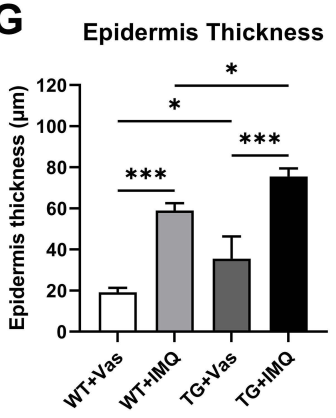

H

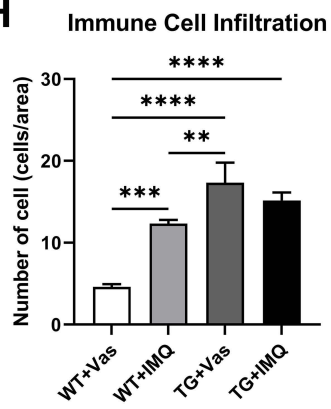

I

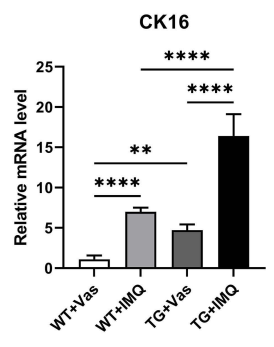

K

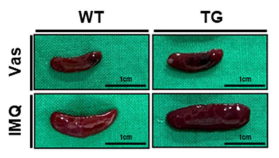

Spleen/Body weight

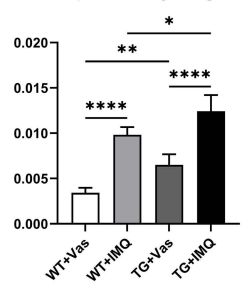

Figure 4 Promoted proliferation of keratinocytes in established Lin28a TG mice: (A) PCR was performed to identify Lin28a overexpressing mice. Primers pAcGFP.NI.F and Lin28a.R were used to detect the exogenous gene for PCR genotyping; (B) total RNAs were isolated from mouse skin tissue and reverse transcribed. Lin28a mRNA expression levels in the skin were analyzed via RT-PCR. Tissue samples were collected from 8-12-week-old mice $(n=6-7)$; (C) overexpressed protein levels were confirmed through Western blotting in mouse skin tissue; (D) total RNAs were isolated from mouse skin tissue and reverse transcribed using TaqMan MicroRNA Reverse Transcription Kit. Relative expression levels of let-7 microRNAs were measured by real-time PCR using TaqMan ${ }^{\mathrm{TM}}$ MicroRNA Assay. T-tests were performed using GraphPad Prism 9. All data are presented as mean \pm SEM; $* p<0.05$, **p $<0.01$, *** $p<0.001$, and $* * * * p<0.0001$ compared with WT mice. WT and Lin28a TG mice were treated with a daily topical dose of $62.5 \mathrm{mg}$ of IMQ cream (5\%) for 5 consecutive days and control mice were treated with the same dose of Vas for 5 consecutive days: (E) phenotypic presentation of mouse back skin for WT and Lin28a TG mice treated with IMQ or Vas for 5 days; (F) light microscopy examination of skin sections stained with H\&E. Scale bars represent $128 \mu \mathrm{m}$; (G) skin thickness was measured in the epidermis of WT and Lin28a TG mice treated with IMQ or Vas for 5 days; (H) immune cell infiltration was measured by counting the cells stained in the dermis from the results of H\&E staining; (I) total RNAs were isolated from mouse skin tissue and reverse transcribed. Relative mRNA expression levels of CKI6, proliferation marker, were analyzed by RT-PCR; (J) light microscopy examination of skin sections stained with Lin28a or Ki67 antibody, scale bars $=128 \mu \mathrm{m}$; $(\mathbf{K})$ phenotypic presentation of mouse spleen and spleen/body weight for WT and Lin28a TG mice treated with IMQ or Vas for 5 days. Two-way ANOVA followed by Tukey's multiple comparisons test was performed using GraphPad Prism 9 . All data are presented as mean \pm SEM; $* p<0.05$, ** $p<0.01$, $* * * p<0.001$, and $* * * * p<0.0001$ compared with each group. 
markers was identified. The expression levels of CK16 was higher in Lin28a TG than in WT; untreated Lin28a TG was higher than untreated WT, and IMQ-treated Lin28a TG was higher than IMQ-treated WT (Figure 4I). Microscopic examination of Ki67-stained skin sections from Lin28 TG and WT mice revealed that the increased expression of Ki67 in Lin28 TG mice indicated accelerated keratinocyte proliferation (Figure 4J). Although CK16 mRNA expression in psoriasislike Lin28a TG was higher than that in psoriasis-like WT, there was no difference in the protein expression of Ki67, which was confirmed by immunohistochemistry. In agreement with the literature, the spleens of IMQ-treated mice became swollen. ${ }^{17}$ However, the spleens of Lin28 TG mice were significantly larger than those of WT mice without IMQ treatment (Figure 4H). In combination, these data indicated that Lin28a accelerated the clinical manifestations of psoriasis.

\section{Lin28a Induces Abnormal Differentiation in Lin28a TG Mice by Regulating Cytokines and Signaling Pathways}

To confirm that the increased proliferation in Lin28a TG mice and the abnormal differentiation and cytokine increase in Lin28a overexpressing $\mathrm{HaCaT}$ cells in the previous figures (Figures 1 and 4) was due to the regulation of let-7 by Lin28a, the expression of let-7 was confirmed when psoriasis was induced in WT and Lin28a TG mice. In this result, WT and Lin28a TG mice had decreased expression of let-7 when psoriasis was induced, and the psoriasis-like TG showed a lower let-7 expression compared with the psoriasis-like WT mice (Figure 5A). To investigate the potential role of Lin28a in the pathogenesis of psoriasis, differentiation markers were used to compare the expression levels of Lin28a TG and WT mice using qRT-PCR of the skin tissue. The results showed an increase in early and late differentiation markers in Lin28a TG + Vas than WT + Vas. After induction by IMQ, mRNA expression of CK1, CK10, and filaggrin were higher in Lin28a TG mice compared with WT. However, the expression of involucrin in the epidermis of Lin28a TG mice was not significantly different in WT mice skin. CK1 increased in Lin28a TG mice compared with untreated TG mice when psoriasis was induced, and the other differentiation markers did not change significantly when psoriasis was induced in Lin28a TG mice compared with untreated TG mice (Figure 5B). In addition, changes in the differentiation markers, which were analyzed using immunohistochemistry of the mice skin (Figure 5C), suggested that Lin28a might have a relationship with psoriasis by enhancing the differentiation of keratinocytes.

Cytokines lead to abnormal keratinocyte maturation and the activation of dendritic cells. ${ }^{18}$ Stressed keratinocytes release IL-6, which recruits macrophages and neutrophils to the inflammation sites. Previous studies revealed that IL-6 is a direct target of let-7b and IL-6 mediated differentiation via signaling cascades, which include ERK, STAT3, and NF- $\mathrm{BB}$ signaling. ${ }^{13}$ To elucidate if Lin28a promoted the differentiation of keratinocytes via ERK, STAT3, and NF- $\mathrm{B}$ by regulating IL-6, psoriasis-related cytokines and chemokines, IL-6, TNF$\alpha$, IL-17A, IL-36 $\gamma$, IL-1 $\beta$, and S100A9, were evaluated. Some of these cytokines and chemokines increased after IMQ treatment in WT mice. The mRNA levels of all these cytokines and chemokines were higher in Lin28a TG mice + Vas compared with WT mice + Vas. For IL6, IL-36 $\gamma$, and S100A9, psoriasis-like Lin28a TG mice through IMQ showed higher expression than IMQtreated WT mice (Figure 5D). In addition, the phosphorylation of signaling was examined using Western blotting. Lin28a overexpression increased phosphorylation of ERK, STAT3, and NF- $\kappa \mathrm{B}$ in nontreated mice. The induction of psoriasis through IMQ increased ERK, STAT3, and NF- $\mathrm{B}$ in WT and Lin28a TG mice. However, the signaling pathways in which Lin28a TG mice with IMQ was highly expressed compared with WT with IMQ were ERK and STAT3 (Figure 5E). These results show that the overexpression of Lin28a upregulated cytokines, which play an important role in psoriasis, and aggravated psoriasis through the phosphorylation of ERK and STAT3.

\section{Discussion}

Psoriasis might be a manifestation of impaired communication between keratinocytes and immune cells that are caused by genetic and environmental factors, this includes $\mathrm{T}$ cells, dendritic cells, and neutrophils. ${ }^{19,20}$ In psoriasis, $\mathrm{T}$ cell infiltration into the dermis and epidermal hyperplasia are key histological features. As selfreactive $\mathrm{T}$ cells infiltrate the skin, they secrete proinflammatory cytokines, such as IL-17 and IL-36 $\gamma .^{21-23}$ The IL-17 family of cytokines induce proliferation and differentiation in keratinocytes. During the early phase of the pathogenic cascade, IL-17 drives a feed-forward inflammatory loop. In response to these cytokines, keratinocytes secrete chemokines (CCL19, CCL20, CXCL1-3, CXCL9, and CXCL10), which recruit 
A

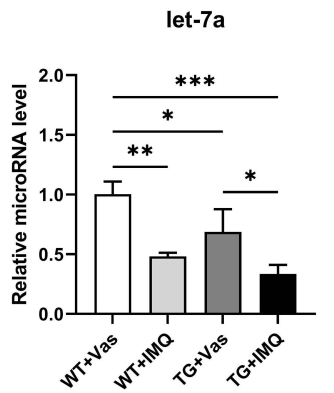

C

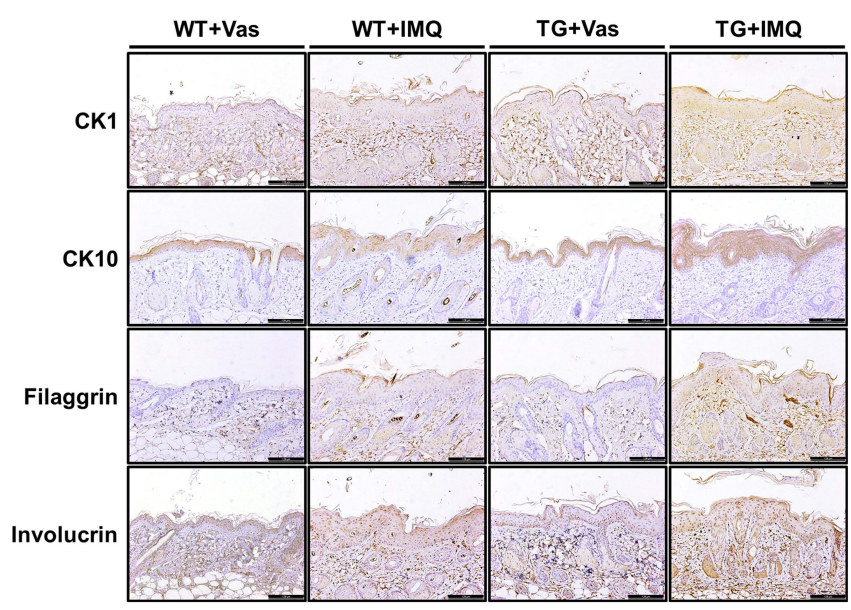

E

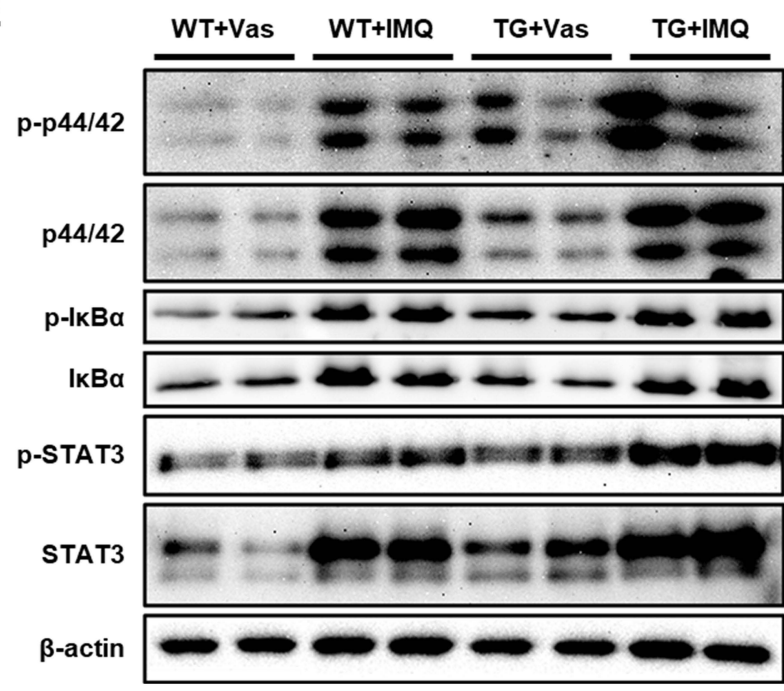

B
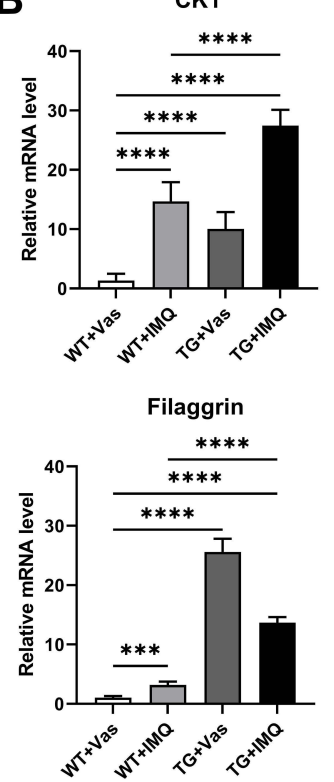

D

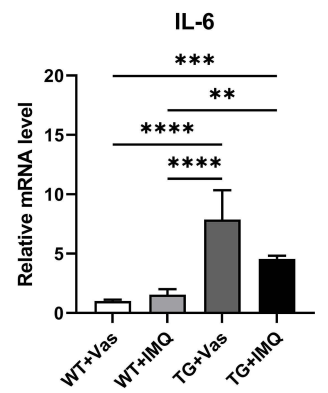

IL-17A

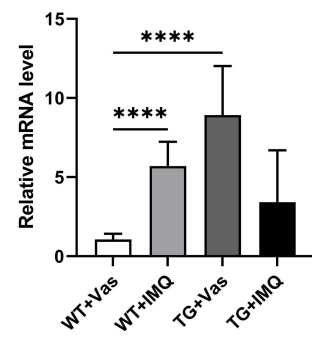

IL-1 $\beta$

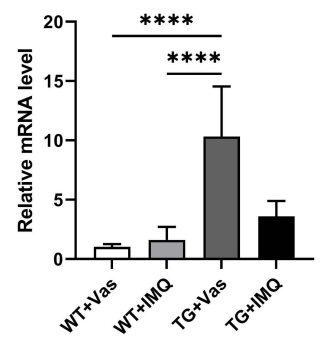

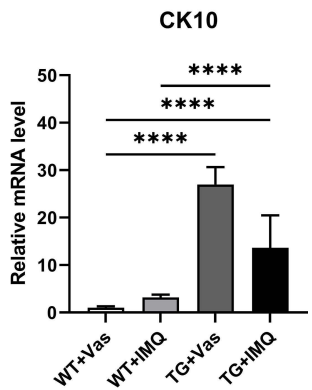
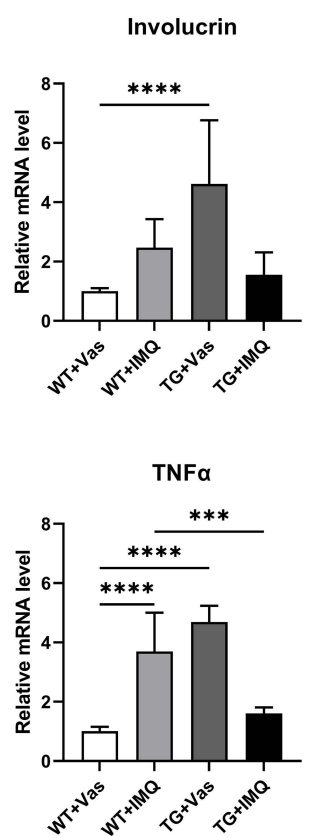

IL-36y

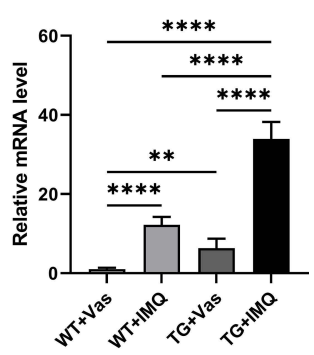

S100A9

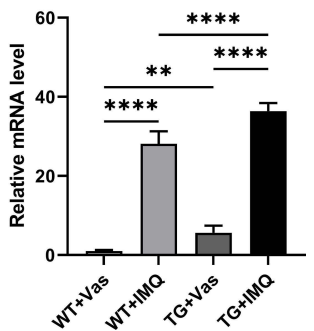

Figure 5 Lin28a induces abnormal differentiation in Lin28a TG mice by regulating cytokines and signaling pathways: (A) total RNAs were isolated from mouse skin tissue and reverse transcribed using TaqMan MicroRNA Reverse Transcription Kit (Applied Biosystems). Relative expression levels of let-7 microRNAs were examined through RT-PCR using TaqMan MicroRNA Assay (Applied Biosystems); (B) total RNAs were isolated from mouse skin tissue and reverse transcribed. Relative mRNA expressions of early and late differentiation markers were analyzed by RT-PCR; (C) skin samples of WT and Lin28a TG with Vas or IMQ were stained with CKI, CKI0, filaggrin, and involucrin. Scale bars represent I $28 \mu$ m; (D) relative mRNA expression of cytokines and chemokines, which play important roles in psoriasis were examined by qRT-PCR; (E) Western blotting analysis of phosphorylated ERK and

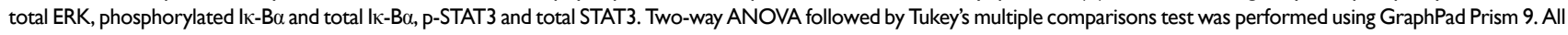
data are presented as mean \pm SEM; ${ }^{*} p<0.05$, ${ }^{* *} p<0.01$, ${ }^{* * *} p<0.001$, and $* * * * p<0.0001$ compared with each group. 
neutrophils, IFN- $\gamma$, or IL-17 producing $\mathrm{T}$ cells, and mature dendritic cells. ${ }^{10,19,24}$ Then, these highly inflammatory skin-infiltrating dendritic cells cause the activation of autoreactive $\mathrm{T}$ cells, and therefore, boost inflammation. $^{24}$

Recently, the role of keratinocytes in psoriasis has become an important research focus. The predominant cell type in human skin is keratinocytes, ${ }^{25}$ which are a major source of inhibitory cytokines. These cytokines enable the skin to remain in an inflammatory quiescent state with no triggers, which would otherwise activate local dendritic cells. ${ }^{26}$ However, an imbalance between the anti-inflammatory and proinflammatory signals might cause the development of inflammatory skin diseases, such as psoriasis and atopic dermatitis. ${ }^{27}$ In this study, we focused on the imbalance of proliferation and differentiation of keratinocytes as the reason for the exacerbation of psoriasis.

Other studies identified the genome association and an increasing number of genetic markers for psoriasis susceptibility. ${ }^{10}$ However, only one-quarter of the heritability of psoriasis has been described, which suggests that there are susceptibility factors that have not been discovered. Because most of the loci that have been identified are in non-coding DNA, genetic variations in functional RNAs, such as microRNAs, might contribute to psoriasis susceptibility. In other studies, let-7b directly acted on IL-6, which affected keratinocyte differentiation through ERK signaling. ${ }^{13}$ Therefore, from the results of this study, Lin28, which is the RNA-binding protein that post-transcriptionally inhibits the biogenesis of let-7 miRNAs, might be instrumental in the pathogenesis of psoriasis. In this study, the results were confirmed by in vitro and in vivo analyses in keratinocytes and TG mice, respectively, that Lin28a plays a pivotal role in keratinocytes during psoriasis.

Wound healing and the pathogenesis of psoriasis have similar characteristics, in particular, with the inflammatory response, keratinocyte proliferation, and proliferation and differentiation of vascular endothelial cells. ${ }^{28}$ Skin transition involves a series of programmed changes that convert basal keratinocytes into anucleate corneocytes. This transformation occurs for approximately 50 days in healthy skin: however, it only requires 5 days to form on lesional psoriatic skin. The epidermis is a dynamic tissue that is composed primarily of keratinocytes that undergo a tightly controlled differentiation process when they migrate from the basal to suprabasal layers. ${ }^{29}$ In the epidermal spinous layer, keratinocytes express CK1 and CK $10 .^{30}$ Therefore, the expressions of $\mathrm{CK} 1$ and $\mathrm{CK} 10$ are the first signs of differentiation. During keratinization, additional proteins (eg, filaggrin and involucrin) are required to form corneodesmosomes and cornified envelopes. ${ }^{3}$ In addition, in this study, the results revealed an increase in proliferation and the expression of CK1, CK10, filaggrin, and involucrin in Lin28a-overexpressing keratinocytes and TG mice compared with Mocks and untreated WT mice. This suggested that the abnormal increase in the proliferation and differentiation of keratinocytes when Lin28a was overexpressed had a significant effect on keratinogenesis, which is one of the hallmarks of psoriasis.

IL-36 $\alpha$ and IL-36 $\gamma$ were identified as being overexpressed in psoriatic lesions. ${ }^{31,32}$ In addition, overexpression of IL-36 cytokine in mice revealed a psoriasis-like pathology of the skin and IL-36 treatment of keratinocytes induced proinflammatory signaling. ${ }^{33}$ The results after IL$36 \gamma$ treatment showed an increased expression of early and late differentiation and proinflammatory cytokines in the Lin28a-overexpressing cell line (Figure 2A and B). In addition, IL-36 $\gamma$ was highly expressed in the skin of Lin28a TG mice (Figure 5D).

For the in vivo experiments, IMQ cream, which is a TLR7 agonist that induces skin inflammation, was applied in a mouse model to study human psoriasis. ${ }^{16}$ The results confirmed that WT mouse skin has higher Lin28a levels combined with a decrease in let-7 after psoriasis inducement by IMQ treatment, which meant that Lin28a might affect psoriasis (Figure 3). Proliferation and differentiation markers increased with the increase in Lin28a expression levels (Figure 3). In psoriasis-induced Lin28a TG mice, epidermal thickness and spleen size were larger than those of WT with IMQ mice (Figure $4 \mathrm{G}$ and $\mathrm{K}$ ). In agreement with the results from $\mathrm{HaCaT}, \mathrm{CK} 1$, and filaggrin mRNA expression increased in psoriasis-induced Lin28a-overexpressing mice compared with WT + IMQ and TG + Vas mice. Although the mRNA level of involucrin decreased after psoriasis inducement in WT and Lin28a TG mice, the immunohistology results showed higher protein levels of involucrin in psoriasis-like inflammation. Of note, CK10 mRNA and the protein levels of Lin28a-overexpressing mice decreased after psoriasis inducement. This result could be explained in previous other studies where CK10 in normal skin cells was lower or had no difference compared with psoriasis patient's keratinocytes. 
Stressed keratinocytes release IL-6, which mobilizes macrophages and neutrophils to the site of inflammation. ${ }^{34}$ In addition, IL-6 mediates differentiation through several signaling cascades, which includes ERK1/2, STAT3, and NF- $\mathrm{kB}$ signaling. In addition, let-7b directly regulates IL-6, which is a strong mediator of a host's response to tissue damage and infection and is produced by keratinocytes and leukocytes. The expression levels of IL-6 in Lin28a-overexpressing $\mathrm{HaCaT}$ and Lin28a TG mice increased combined with an increase in other psoriasis-associated proinflammatory cytokines (TNF- $\alpha$, IL-1 $\beta$, IL-36 $\gamma$, and IL-17A) and chemokines (S100A9). Therefore, the characteristic cytokine and chemokines of psoriasis, IL-36 $\gamma$ and S100A9, were highly expressed in nontreated Lin28a TG, and when psoriasis was induced, they increased compared with psoriasis-like WT mice. However, other proinflammatory cytokines (TNF- $\alpha$, IL-1 $\beta$ ) and IL-17 did not show a significant increase in psoriasislike TG mice compared with psoriasis-like WT mice (Figure 5D). As mentioned earlier, let-7 targets and inhibits IL- $6 .{ }^{13}$ In addition, according to other studies, let-7 inhibits the differentiation of IL-6-dependent Th17 cells. ${ }^{35}$ According to this study, it was not surprising that the secretion of IL-6 and IL-17 increases when let-7 expression is suppressed by Lin28a overexpression. However, if psoriasis was induced by IMQ in a state when IL-6 and IL-17 was already high due to Lin28a overexpression, the results might be different. The induction of psoriasis by IMQ was achieved by regulating various pathways, which included TLR7/8 and NLRP3/NALP3 or adenosine receptors. Although IMQ is the most widely used psoriasis modeling method, it remains unclear how the cytokines, which are already elevated in Lin28a TG mice due to Lin28a overexpression, are regulated by IMQ. Therefore, in this study, changes in the proliferation and differentiation of keratinocytes in the skin rather than on cytokines were focused on.

For the mechanisms of Lin28a in keratinocytes, the downstream signaling pathways of IL-6, ERK1/2, STAT3, and NF- $\mathrm{KB}$ signaling were analyzed (Figure 5E). ERK1/2 phosphorylation has been demonstrated in lesioned psoriatic skin compared with non-lesioned psoriatic skin. In agreement with previous reports, Lin28a-overexpressing mice skin showed stronger ERK1/2 phosphorylation, and the total and phosphorylated forms of p44/42 ERK1/2 were stronger in IMQ-treated mouse skin. In addition, the activation of STAT3 signaling that was measured by Western blotting increased in Lin28a TG mice compared with WT mice. In the case of STAT3, Lin28a overexpressing TG mice showed increased basal levels as well as phosphorylated levels (Figure 5E). From other research, STAT3 is a target of let-7b, and let-7b negatively regulates STAT3 mRNA and protein expression levels. ${ }^{36}$ According to this research, the increase in basal levels of STAT3 expression might be due to let-7b suppression by Lin28a overexpression. However, NF$\kappa \mathrm{B}$ signaling was hardly different in WT and TG after psoriasis induction with IMQ (Figure 5E). This result was thought to be due to the psoriasis induction pathway of IMQ. IMQ induces psoriasis by regulating TLR7/8 and its downstream signals. TLR7/8, which is stimulated by IMQ, amplifies NF- $\mathrm{kB}$ through MyD 88, which results in the increased secretion of inflammatory cytokines. Therefore, $\mathrm{NF}-\mathrm{\kappa B}$ activation increased when IMQ was treated in WT and TG mice in this result. In addition, Lin28a overexpression activates IL-6-dependent NF- $\mathrm{KB}$ by regulating let-7. However, the activation of NF- $\mathrm{BB}$ was not higher in TG with IMQ compared with TG with IMQ, which could be due to the regulation of NF- $\mathrm{KB}$ by Lin28a and the regulation of NF- $\kappa B$ IMQ conflicted with each other. In combination, the overexpression of Lin28a enhanced the expression of inflammatory cytokines, especially IL-6, that led to acanthosis and disease severity by the activation of ERK and STAT3 signaling. According to the literature, STAT3 is a target of let-7b and let-7b negatively regulates STAT3 mRNA and protein expression levels. ${ }^{37}$

In this study, the overexpression of Lin28a in TG mice led to increased proliferation and differentiation via ERK1/ 2 and STAT3 signaling, and aggravated disease severity in the psoriasis mouse model.

\section{Acknowledgments}

This research was supported by the Basic Science Research Program through the National Research Foundation of Korea (NRF) funded by the Ministry of Education (2020R111A2075315, 2020R1I1A1A01074542, and 2020R1A4A1018280).

\section{Disclosure}

The authors report no conflicts of interest in this work.

\section{References}

1. Krueger G, Ellis CN. Psoriasis-recent advances in understanding its pathogenesis and treatment. $J$ Am Acad Dermatol. 2005;53(1 Suppl 1): S94-S100. doi:10.1016/j.jaad.2005.04.035

2. Bhosle MJ, Kulkarni A, Feldman SR, Balkrishnan R. Quality of life in patients with psoriasis. Health Qual Life Outcomes. 2006;4:35. doi:10.1186/1477-7525-4-35 
3. Bovenschen HJ, Seyger MM, Van de Kerkhof PC. Plaque psoriasis vs. atopic dermatitis and lichen planus: a comparison for lesional T-cell subsets, epidermal proliferation and differentiation. $\mathrm{Br} \mathrm{J}$ Dermatol. 2005;153(1):72-78. doi:10.1111/j.1365-2133.2005.06538.x

4. Benhadou F, Mintoff D, Del Marmol V. Psoriasis: keratinocytes or immune cells - which is the trigger? Dermatology. 2019;235 (2):91-100. doi:10.1159/000495291

5. Chandra A, Ray A, Senapati S, Chatterjee R. Genetic and epigenetic basis of psoriasis pathogenesis. Mol Immunol. 2015;64(2):313-323. doi:10.1016/j.molimm.2014.12.014

6. Muller A, Hennig A, Lorscheid S, et al. IkappaBzeta is a key transcriptional regulator of IL-36-driven psoriasis-related gene expression in keratinocytes. Proc Natl Acad Sci U S A. 2018;115 (40):10088-10093. doi:10.1073/pnas.1801377115

7. Shyh-Chang N, Daley GQ. Lin28: primal regulator of growth and metabolism in stem cells. Cell Stem Cell. 2013;12(4):395-406. doi:10.1016/j.stem.2013.03.005

8. Ustianenko D, Chiu HS, Treiber T, et al. LIN28 selectively modulates a subclass of let-7 MicroRNAs. Mol Cell. 2018;71(2):271-83e5. doi:10.1016/j.molcel.2018.06.029

9. Hawkes JE, Nguyen GH, Fujita M, et al. microRNAs in psoriasis. J Invest Dermatol. 2016;136(2):365-371. doi:10.1038/JID.2015.409

10. Liu Y, Liu Q. MicroRNAs as regulatory elements in psoriasis. Open Med. 2016;11(1):336-340. doi:10.1515/med-2016-0063

11. Pasquinelli AE, Reinhart BJ, Slack F, et al. Conservation of the sequence and temporal expression of let-7 heterochronic regulatory RNA. Nature. 2000;408(6808):86-89. doi:10.1038/35040556

12. Zhao C, Sun G, Li S, et al. MicroRNA let-7b regulates neural stem cell proliferation and differentiation by targeting nuclear receptor TLX signaling. Proc Natl Acad Sci U S A. 2010;107(5):1876-1881. doi:10.1073/pnas.0908750107

13. Wu Y, Liu L, Bian C, et al. MicroRNA let-7b inhibits keratinocyte differentiation by targeting IL-6 mediated ERK signaling in psoriasis. Cell Commun Signal. 2018;16(1):58. doi:10.1186/ s12964-018-0271-9

14. Albanesi C, Madonna S, Gisondi P, Girolomoni G. The interplay between keratinocytes and immune cells in the pathogenesis of psoriasis. Front Immunol. 2018;9:1549. doi:10.3389/fimmu.2018.01549

15. Nestle FO, Kaplan DH, Barker J. Psoriasis. N Engl J Med. 2009;361 (5):496-509. doi:10.1056/NEJMra0804595

16. Wagner EF, Schonthaler HB, Guinea-Viniegra J, Tschachler E. Psoriasis: what we have learned from mouse models. Nat Rev Rheumatol. 2010;6(12):704-714. doi:10.1038/nrrheum.2010.157

17. Qin S, Wen J, Bai XC, et al. Endogenous n-3 polyunsaturated fatty acids protect against imiquimod-induced psoriasis-like inflammation via the IL-17/IL-23 axis. Mol Med Rep. 2014;9(6):2097-2104. doi: $10.3892 / \mathrm{mmr} .2014 .2136$

18. Grossman RM, Krueger J, Yourish D, et al. Interleukin 6 is expressed in high levels in psoriatic skin and stimulates proliferation of cultured human keratinocytes. Proc Natl Acad Sci U S A. 1989;86 (16):6367-6371. doi:10.1073/pnas.86.16.6367

19. Mak RK, Hundhausen C, Nestle FO. Progress in understanding the immunopathogenesis of psoriasis. Actas Dermosifiliogr. 2009;100 (Suppl 2):2-13. doi:10.1016/s0001-7310(09)73372-1

20. Srivastava A, Nikamo P, Lohcharoenkal W, et al. MicroRNA-146a suppresses IL-17-mediated skin inflammation and is genetically associated with psoriasis. J Allergy Clin Immunol. 2017;139(2):550-561. doi:10.1016/j.jaci.2016.07.025

21. Duhen T, Geiger R, Jarrossay D, Lanzavecchia A, Sallusto F. Production of interleukin 22 but not interleukin 17 by a subset of human skin-homing memory $\mathrm{T}$ cells. Nat Immunol. 2009;10 (8):857-863. doi:10.1038/ni.1767
22. Becher B, Pantelyushin S. Hiding under the skin: interleukin-17producing $\gamma \delta \mathrm{T}$ cells go under the skin? Nat Med. 2012;18 (12):1748-1750. doi:10.1038/nm.3016

23. Kagami S, Rizzo HL, Lee JJ, Koguchi Y, Blauvelt A. Circulating Th17, Th22, and Th1 cells are increased in psoriasis. J Invest Dermatol. 2010;130(5):1373-1383. doi:10.1038/jid.2009.399

24. Orlik C, Deibel D, Kublbeck J, et al. Keratinocytes costimulate naive human T cells via CD2: a potential target to prevent the development of proinflammatory Th1 cells in the skin. Cell Mol Immunol. 2020;17 (4):380-394. doi:10.1038/s41423-019-0261-x

25. Li J, Li X, Hou R, et al. Psoriatic T cells reduce epidermal turnover time and affect cell proliferation contributed from differential gene expression. J Dermatol. 2015;42(9):874-880. doi:10.1111/13468138.12961

26. Sharma A, Saito Y, Hung SI, Naisbitt D, Uetrecht J, Bussiere J. The skin as a metabolic and immune-competent organ: implications for drug-induced skin rash. $J$ Immunotoxicol. 2019;16(1):1-12. doi:10.1080/1547691x.2018.1514444

27. Albanesi C, De Pita O, Girolomoni G. Resident skin cells in psoriasis: a special look at the pathogenetic functions of keratinocytes. Clin Dermatol. 2007;25(6):581-588. doi:10.1016/j.clindermatol.20 07.08 .013

28. Young PM, Parsi KK, Schupp CW, Armstrong AW. Psoriasis and wound healing outcomes: a retrospective cohort study examining wound complications and antibiotic use. Dermatol Online J. 2017;23(11). doi:10.5070/D32311037238

29. Xia J, Zhang W. MicroRNAs in normal and psoriatic skin. Physiol Genomics. 2014;46(4):113-122. doi:10.1152/physiolgenomics.0 0157.2013

30. Huang KF, Ma KH, Liu PS, Chen BW, Chueh SH. Baicalein increases keratin 1 and 10 expression in HaCaT keratinocytes via TRPV4 receptor activation. Exp Dermatol. 2016;25(8):623-629. doi:10.1111/exd.13024

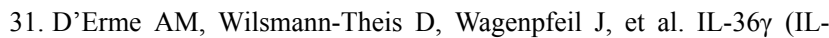
1F9) is a biomarker for psoriasis skin lesions. J Invest Dermatol. 2015;135(4):1025-1032. doi:10.1038/jid.2014.532

32. Boutet MA, Bart G, Penhoat M, et al. Distinct expression of interleukin (IL)-36 $\alpha, \beta$ and $\gamma$, their antagonist IL-36Ra and IL-38 in psoriasis, rheumatoid arthritis and crohn's disease. Clin Exp Immunol. 2016;184(2):159-173. doi:10.1111/cei.12761

33. Mahil SK, Catapano M, Di Meglio P, et al. An analysis of IL-36 signature genes and individuals with IL1RL2 knockout mutations validates IL-36 as a psoriasis therapeutic target. Sci Transl Med. 2017;9:411. doi:10.1126/scitranslmed.aan2514

34. Cai Y, Xue F, Quan C, et al. A critical role of the IL-1beta-IL-1R signaling pathway in skin inflammation and psoriasis pathogenesis. J Invest Dermatol. 2019;139(1):146-156. doi:10.1016/j.jid.2018.07.025

35. Zhang Y, Wang X, Zhong M, Zhang M, Suo Q, Lv K. MicroRNA let-7a ameliorates con A-induced hepatitis by inhibiting IL-6-dependent Th17 cell differentiation. J Clin Immunol. 2013;33 (3):630-639. doi:10.1007/s10875-012-9840-7

36. Hu XP, Xie Q, Chen CF, Zhang W, Yu B. Let-7a inhibits T-cell proliferation and IFN-gamma secretion by down-regulating STAT3 expression in patients with psoriasis. Cell Physiol Biochem. 2017;42 (1):115-125. doi: $10.1159 / 000477120$

37. Hua K, Deng X, Hu J, et al. Long noncoding RNA HOST2, working as a competitive endogenous RNA, promotes STAT3-mediated cell proliferation and migration via decoying of let-7b in triple-negative breast cancer. J Exp Clin Cancer Res. 2020;39(1):58. doi:10.1186/ s13046-020-01561-7 


\section{Publish your work in this journal}

The Journal of Inflammation Research is an international, peerreviewed open-access journal that welcomes laboratory and clinical findings on the molecular basis, cell biology and pharmacology of inflammation including original research, reviews, symposium reports, hypothesis formation and commentaries on: acute/chronic inflammation; mediators of inflammation; cellular processes; molecular mechanisms; pharmacology and novel anti-inflammatory drugs; clinical conditions involving inflammation. The manuscript management system is completely online and includes a very quick and fair peerreview system. Visit http://www.dovepress.com/testimonials.php to read real quotes from published authors. 